\title{
Impact of Probe Configurations on Maximum of Test Volume Size in 3D MIMO OTA Testing
}

\author{
Weimin Wang, Huaqiang Gao, Yongle Wu, and Yuanan Liu \\ The Beijing Key Laboratory of Work Safety Intelligent Monitoring, School of Electronic Engineering, Beijing University of \\ Posts and Telecommunications, Beijing 100876, China
}

Correspondence should be addressed to Yongle Wu; wuyongle138@gmail.com

Received 16 October 2017; Accepted 5 December 2017; Published 26 December 2017

Academic Editor: Lei Ge

Copyright (C) 2017 Weimin Wang et al. This is an open access article distributed under the Creative Commons Attribution License, which permits unrestricted use, distribution, and reproduction in any medium, provided the original work is properly cited.

With the development of multiple-input-multiple-output (MIMO) technology, the over-the-air (OTA) testing of MIMO capable devices with different sizes needs to be conducted for performance evaluation. The device under test (DUT) should be within a tridimensional test volume created by multiprobe configurations. Thus, determining the maximum size of test volume could be vital to test the DUT of different size and larger test volumes should be adopted to evaluate larger DUTs. All types of probe configurations including the fixed and the flexible probe configurations are investigated in this paper to address this issue. The maximum of test volume size (MTVS) is determined within the given error threshold of spatial correlation for a given probe configuration. Simultaneously, the impact of different probe configurations on MTVS is studied in order to obtain larger MTVSs. Simulation results show that larger MTVSs can be obtained by utilizing the optimal probe configuration with any given 3D channel model for 3D MIMO OTA testing.

\section{Introduction}

Multiple-input-multiple-output (MIMO), an important technology of high-speed wireless communication, has been adopted in Long Term Evolution (LTE) and LTE-Advanced [1]. Massive MIMO systems employing a large number of antennas are used in radio base stations (BSs). As a multiuser MIMO technology, it would be applied to the fifth-generation (5G) mobile communication system in the future [2]. MIMO capable devices include user equipment (UE) assembled with several antennas and massive MIMO BS equipped with hundreds of antennas. In order to evaluate the performance of MIMO capable devices, they need to be tested under realistic channel propagation environment in the laboratory. MIMO over-the-air (OTA) testing [3] has been considered as a promising method for evaluation of the performance of MIMO capable devices by 3GPP, CTIA, and COST. Among the several MIMO OTA testing methods, the MIMO OTA testing method based on multiprobe anechoic chamber (MPAC) becomes one of the most competitive methods for its ability to reproduce the multipath wireless channel environment and obtain higher emulation precision [4].
Three main modules are involved in the MPAC method, namely, BS emulator or UE emulator (BS emulator adopted for UE testing and UE emulator adopted for massive BS testing), channel emulator, and anechoic chamber. The anechoic chamber is mainly composed of multiprobe setup, absorbers and device under test (DUT). The multiprobe setup is distributed with multiple probes, each of which includes two polarized antennas (i.e., vertical polarization and horizontal polarization); the absorbers could attenuate the electromagnetic waves projected on their surface greatly and eliminate the reflected electromagnetic waves; the DUT is placed in the center of the anechoic chamber with the multiprobe setup. An ideal realistic communication environment for the DUT could be created by the multiprobe setup and absorbers. The MPAC method could provide a tridimensional test volume around the DUT where the performance test of DUT can be imitated in the realistic channel environment. Note that the DUT size is less than the test volume.

The MPAC method includes two different dimensions of condition due to the different channel models, namely, two-dimensional (2D) and three-dimensional (3D). Most of the standard channel models are 2D channel models; that 
is, only horizontal azimuth angles are defined without the extension of vertical elevation angles. Some of the literatures adopted the 2D MPAC method to emulate a $2 \mathrm{D}$ standard channel model in a 2D multiprobe setup [5-7], in which eight probes are placed horizontally on a probe ring. However, the assumption of a $2 \mathrm{D}$ channel model is not sufficient to reproduce the realistic channel environment because the elevation angle cannot be neglected in the propagation environment. Hence, an accurate realistic channel propagation environment should be reproduced when an elevation extension is introduced. Reference [8] extends the 2D MPAC method to the 3D MPAC method, in which the multiprobe setup is $3 \mathrm{D}$ and the probes are distributed on a sphere. These probes compose several probe planes of different elevations where the probes are placed evenly in a circle on each plane, that is, probe circle. Taking sixteen probes as an example, there are three probe circles including the upper, middle, and lower one. Eight probes are on the middle probe circle, while four probes are on the upper one and the other on the lower one, respectively.

The channel environment in the test volume is mostly generated by geometry-based stochastic models (GBSM), which introduce the concept of clusters, each of which has a specific angle power spectrum. The prefaded signal synthesis (PFS) technique reproduces the channel spatial characteristics in the test volume as a channel emulation technique [9]. Meanwhile, it generates a prefaded signal in each probe of the multiprobe setup based on the angular power spectrum of the cluster. The prefaded signals that could synthesize the target channel environment in the test volume are generated by assigning the corresponding power weights to the respective probes. References $[10,11]$ apply the PFS technique to the 3D MPAC method. The power spectrum of the cluster is extended from the original 2D power azimuth spectrum (PAS) to a $3 \mathrm{D}$ spherical power spectrum (SPS). Different 3D channel models have variable SPSs, and different SPSs produce altered 3D channel spatial characteristics. The spatial correlation is utilized frequently as a figure of merit (FoM) to evaluate the $3 \mathrm{D}$ channel spatial characteristics. Thus, the error between the target and emulated spatial correlation is employed to measure the reconstruction accuracy of the $3 \mathrm{D}$ channel emulation.

Several contributions have reported that 3D channel spatial characteristics are reconstructed in 3D multiprobe setups for the UE testing. Four different diameters of spherical test volume are compared to obtain the maximum diameter in [10]. However, the test volume is sampled on the three axes, which might lead to the bad reconstruction accuracy at certain locations. In other words, the maximum diameter is just considered for three directions and not exact for all directions. In [8], the test volume is sampled by selecting locations on the surface of the ellipsoid, but the size of test volume is fixed to a certain value, without considering how to determine the maximum size of test volume within given accepted correlation error. If the maximum of test volume size (MTVS) for DUT can be determined in spherical multiprobe setups, the MIMO terminals of different size can be evaluated in the anechoic chamber. A few works have also reported the OTA evaluation of massive MIMO BSs where a sectored
MPAC configuration is proposed [12]. Compared with the $\mathrm{UE}$, the electrical size of the massive MIMO BS is much larger. Hence, larger test volumes are needed to evaluate larger MIMO capable devices. Importantly, the test volume size depends directly on the number of probe antennas and is limited owing to the limited number of probe antennas and output ports of the radio channel emulator [13]. Thus, it becomes significant to increase the test volume size with a limited number of probe antennas. So as to increase MTVS, it would be a desirable solution to assess the impact of different probe configurations on MTVS. However, few contributions have addressed this issue.

In this paper, a novel method is presented to obtain MTVS. The MTVS for DUT is determined in spherical multiprobe setups. The test volume is sampled on the surface of a sphere. Meanwhile, this paper investigates the impact of probe configurations on MTVS to obtain larger MTVSs. The probe configurations include the fixed probe configuration and the flexible probe configurations. The fixed probe configuration means that there are several probe circles in the probe setup, each of which is with probes placed uniformly. The flexible probe configuration stands for that the probes of the setup are distributed arbitrarily for both elevation angles and azimuth angles. The fixed probe configuration is adopted to consider the effect of azimuth and elevation angles independently. The flexible probe configuration is employed to study the effect of both azimuth and elevation angles simultaneously. Moreover, the effect of number of probes is discussed in the flexible configuration as well.

The aim of this paper is multifold:

(1) The MTVS can be determined by calculated results of spatial correlation error directly and effectively without complicated system validation and electromagnetic calculation [14]. The maximum of DUT size can be achieved for a given probe configuration.

(2) Different probe configurations including the fixed and the flexible probe configurations are assessed for MTVS. The azimuth and elevation angles are varied independently for the fixed probe configuration, while the azimuth and elevation angles are changed simultaneously for the flexible probe configuration. In the flexible probe configuration, two kinds of optimization algorithms are combined to acquire the optimal probe configuration for larger MTVSs than those in the fixed probe configuration.

(3) The proposed method that could determine and increase the MTVS provides a general framework to decide MTVS for any channel model and probe configuration. Simultaneously, larger MTVSs for any channel model can be obtained with the optimal probe configuration.

\section{Method}

2.1. 3D Spherical Power Spectrum. The 3D spherical power spectrum (SPS) could be modeled as a function of both elevation angle $(\theta)$ and azimuth angle $(\phi)$. It consists of power 


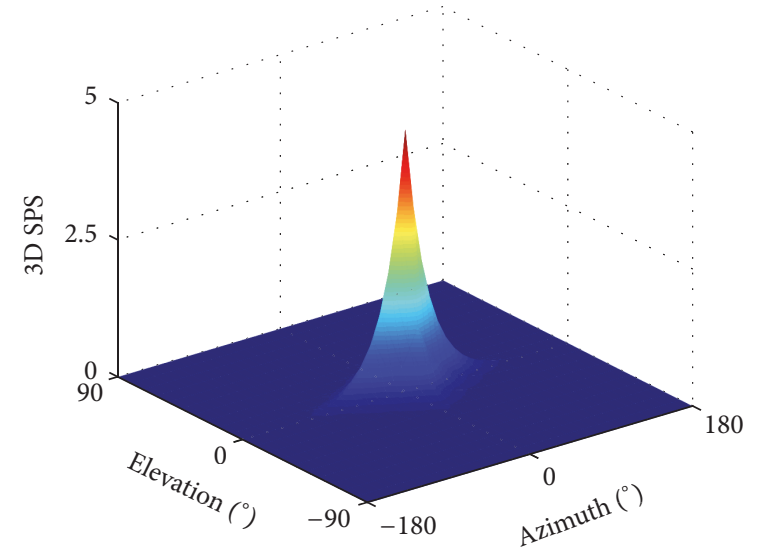

(a) SPS scenario A

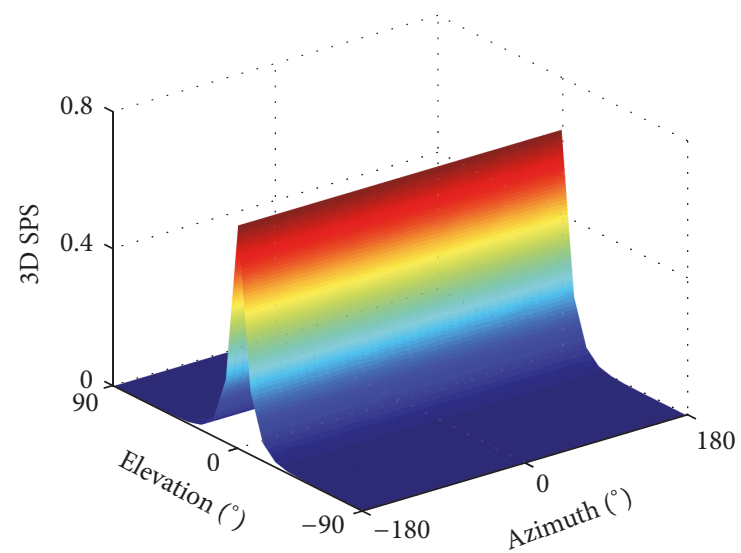

(b) SPS scenario B

FIgURE 1: Two considered SPS scenarios.

elevation spectrum (PES) and power azimuth spectrum (PAS) [15]:

$$
\operatorname{SPS}(\Omega)=\operatorname{SPS}(\theta, \phi)=\operatorname{PES}(\theta) \operatorname{PAS}(\phi) .
$$

where $\Omega$ is the solid angle. The PES and PAS are functions of $\theta$ and $\phi$, respectively. Moreover, the SPS satisfies the condition of $\oint \operatorname{SPS}(\Omega) \mathrm{d} \Omega=1$.

The PAS represents the power distribution of different incoming waves in the horizontal azimuth, and PES denotes the power distribution of the different incoming waves at the vertical height. The PAS is characterized by azimuth of arrival (AoA) and azimuth spread (AS), while the PES is characterized by elevation of arrival (EoA) and elevation spread (ES). AoA and EoA are the mean values of the angles of the different incoming waves in the horizontal azimuth and at the vertical height, respectively, and AS and ES represent the standard deviations of the angles of the different incoming waves in the horizontal azimuth and at the vertical height, respectively.

Two target SPS scenarios (A and B) are considered in this paper.

\subsubsection{SPS Scenario A: SPS of Laplacian Distribution for Both $P E S$ and $P A S$}

$$
\begin{array}{rr}
\operatorname{PES}(\theta)=Q_{\theta} \exp \left(-\frac{\sqrt{2}|\theta-\bar{\theta}|}{\sigma_{\theta}}\right), & {\left[-\frac{\pi}{2}, \frac{\pi}{2}\right]} \\
\operatorname{PAS}(\phi)=Q_{\phi} \exp \left(-\frac{\sqrt{2}|\phi-\bar{\phi}|}{\sigma_{\phi}}\right), & {[-\pi, \pi] .}
\end{array}
$$

\subsubsection{SPS Scenario B: SPS of Laplacian Distribution for PES} and Uniform Distribution for PAS

$$
\begin{aligned}
& \operatorname{PES}(\theta)=Q_{\theta} \exp \left(-\frac{\sqrt{2}|\theta-\bar{\theta}|}{\sigma_{\theta}}\right), \quad\left[-\frac{\pi}{2}, \frac{\pi}{2}\right] \\
& \operatorname{PAS}(\phi)=\frac{1}{2 \pi}, \quad[-\pi, \pi],
\end{aligned}
$$

where $\bar{\theta}$ and $\bar{\phi}$ are the mean elevation and azimuth angles of arrival of incoming waves, respectively. $\sigma_{\theta}$ and $\sigma_{\phi}$ are the elevation and azimuth angular spreads, respectively. $Q_{\theta}$ and $Q_{\phi}$ are scaling constants for PES and PAS, respectively, ensuring that $\oint \operatorname{SPS}(\Omega) \mathrm{d} \Omega=1$ is fulfilled. In this paper, it is assumed that mean elevation angle is $0^{\circ}\left(\bar{\theta}=0^{\circ}\right)$ and mean azimuth angle is $0^{\circ}$ as well $\left(\bar{\phi}=0^{\circ}\right)$, while elevation spread (ES) is $10^{\circ}\left(\sigma_{\theta}=10^{\circ}\right)$, and azimuth spread is $35^{\circ}\left(\sigma_{\phi}=35^{\circ}\right)$. The two considered SPS scenarios are illustrated in Figures $1(\mathrm{a})$ and $1(\mathrm{~b})$.

2.2. Criteria to Evaluate MTVS. The spatial correlation error is adopted as a FoM to evaluate the test volume size of 3D MIMO OTA setups in this paper. The spatial correlation at DUT antenna elements $u$ and $v$ can be expressed as follows [16]:

$$
\begin{aligned}
& \rho_{a} \\
& =\frac{\oint \mathbf{F}_{u}(\Omega) \mathbf{F}_{v}^{*}(\Omega) \operatorname{SPS}(\Omega) \mathrm{d} \Omega}{\sqrt{\oint\left|\mathbf{F}_{u}(\Omega)\right|^{2} \operatorname{SPS}(\Omega) \mathrm{d} \Omega} \sqrt{\oint\left|\mathbf{F}_{v}(\Omega)\right|^{2} \operatorname{SPS}(\Omega) \mathrm{d} \Omega}},
\end{aligned}
$$

where ()$^{*}$ denotes complex conjugate operation and $\mathbf{F}_{u}(\Omega)$ and $\mathbf{F}_{v}(\Omega)$ are the complex radiation patterns of DUT antennas $u$ and $v$, respectively, with a common phase center.

Considering the OTA probes are in the far field with respect to the test volume and DUT antenna pattern is isotropic, (4) can be rewritten as [8]

$$
\begin{aligned}
& \rho_{3 \mathrm{D}}(d, m) \\
& \quad=\oint \exp \left(j k\left(\vec{r}_{u, m}-\vec{r}_{v, m}\right) \cdot \vec{\Omega}\right) \operatorname{SPS}(\Omega) \mathrm{d} \Omega,
\end{aligned}
$$

where $\vec{r}_{u, m}$ and $\vec{r}_{v, m}$ are two sample points at the opposite position of the surface of the fixed spherical test volume in the anechoic chamber, which are defined as the $m$ th location pair. The length of $\vec{r}_{u, m}-\vec{r}_{v, m}$ represents the DUT antenna separation $d$, that is, the diameter of spherical test volume. $\vec{\Omega}$ 
is a $3 \mathrm{D}$ unit vector denoting the solid angle $\Omega$. $k$ is the wave number. Note that the diameter $d$ of spherical test volume represents the test volume size. Therefore, the target spatial correlation $\rho_{3 \mathrm{D}}(d, m)$ is the function of the test volume size $d$ for the $m$ th location pair.

In $3 \mathrm{D}$ multiprobe OTA setups with a limited number of antennas $N$, the emulated spatial correlation could be calculated:

$$
\rho_{\text {OTA }}(\mathbf{w}, d, m)=\sum_{n=1}^{N} w_{n} \exp \left(j k\left(\vec{r}_{u, m}-\vec{r}_{v, m}\right) \cdot \vec{\Phi}_{n}\right),
$$

where $\mathbf{w}=\left[w_{1}, \ldots, w_{n}, \ldots, w_{N}\right]^{T}$ is a power weighting vector to be optimized. $\vec{\Phi}_{n}$ is a $3 \mathrm{D}$ unit position vector of the $n$th probe. The total number of probes is defined as $N$. Once the power weighting vector $\mathbf{w}$ is determined, the emulated spatial correlation $\rho_{\text {OTA }}(d, m)$ is only the function of the test volume size $d$ for the $m$ th location pair.

The spatial correlation error $\left(\left|\rho_{3 \mathrm{D}}(d, m)-\rho_{\mathrm{OTA}}(d, m)\right|\right)$ is obtained from the absolute value of the difference between target spatial correlation $\rho_{3 \mathrm{D}}(d, m)$ and emulated spatial correlation $\rho_{\text {OTA }}(d, m)$ for the $m$ th location pair. Hence, the MTVS could be determined according to the root mean square (rms) of the spatial correlation error $\mid \rho_{3 \mathrm{D}}(d)-$ $\rho_{\text {OTA }}(d) \mid$. For $M$ location pairs, the rms error $\sigma_{\text {rms }}(d)$ that is a function of the test volume size $d$ can be expressed as follows:

$$
\sigma_{\mathrm{rms}}(d)=\sqrt{\frac{1}{M} \sum_{m=1}^{M}\left|\rho_{3 \mathrm{D}}(d, m)-\rho_{\mathrm{OTA}}(d, m)\right|^{2}}
$$

The threshold of $0.05[10]$ is given for $\sigma_{\mathrm{rms}}(d)$ to determine the MTVS. Therefore, MTVS can be obtained within a maximum rms error of 0.05 . It should be mentioned that the MTVS is recommended for MIMO OTA testing in the multiprobe anechoic chamber.

2.3. Optimal OTA Antenna Power Weights. The power weighting vector $\mathbf{w}$ in (6) could be determined by convex optimization [17]. The objective function $f_{O}(d)$ of convex optimization is described as

$$
\begin{aligned}
f_{\mathrm{O}}(d)=\min _{\mathbf{w}} & \left\|\boldsymbol{\rho}_{3 \mathbf{D}}(d)-\boldsymbol{\rho}_{\mathrm{OTA}}(\mathbf{w}, d)\right\|_{2}^{2} \\
\text { s.t. } & 0 \leq w_{i} \leq 1 \quad(\forall i \in[1, N]),\|\mathbf{w}\|_{1}=1,
\end{aligned}
$$

where $\boldsymbol{\rho}_{3 \mathrm{D}}(d)$ and $\boldsymbol{\rho}_{\mathrm{OTA}}(\mathbf{w}, d)$ are the target spatial correlation and emulated spatial correlation vectors, respectively, with each element corresponding to the spatial correlation between two isotropic antennas at a certain location pair inside the fixed test volume.

2.4. Probe Location Optimization. Combined with the aforementioned convex optimization, what is called probe location optimization algorithm (PLOA) is adopted to obtain the optimal probe location and probe weights in flexible probe configurations.

The convex optimization and genetic algorithm (GA) are used to obtain the minimal rms error of spatial correlation in a fixed test volume [18]. In [18], the convex optimization is adopted to optimize power weights for each probe once the probe locations are selected in the iterative process of GA. The objective function of convex optimization in (8) is adopted as the fitness function of GA. Therefore, the minimal rms error of spatial correlation is not achieved until the maximum iterative number is reached. The method description in [18] is not detailed here. On the basis of [18], the different test volume sizes are considered in this paper. Differently, the objective in this paper is to minimize the sum of rms errors in different test volume sizes using convex optimization and PLOA. It is clear that different test volume sizes have different objective function values of convex optimization in (8). The sum of objective function values for different test volume sizes is employed as the fitness function $f_{F}$ of PLOA as follows:

$$
f_{F}=\sum_{l=1}^{L} f_{O}\left(d_{l}\right)
$$

where $\mathbf{d}=\left[d_{1}, \ldots, d_{l}, \ldots, d_{L}\right]$ with $d_{l}=d_{l-1}+d_{\text {step }}$ being a vector representing the different test volume sizes. $L$ is the number of test volume sizes. In addition, $d_{\text {step }}$ is the step of $\mathbf{d}$.

Three PLOAs are adopted for comparison in this paper, namely, the GA [19], the chicken swarm optimization (CSO) [20], and the improved chicken swarm optimization (ICSO) [21].

2.4.1. GA. GA is an optimization method for mimicking natural selection and genetic mechanism. It is based on Darwin's theory of biological evolution and Mendel's theory of genetics. Gene hybridization and gene mutations would produce offspring with strong environmental adaptability, and the genetic structure with high fitness is preserved by natural selection of the survival of the fittest. Therefore, GA is formed by imitating the biological genetic, evolutionary principle, and citing the principle of random statistics. Nevertheless, its ability to explore the new space is limited and it can converge to the local optimal solution easily. Moreover, the search speed is relatively slow so that more training time is needed to obtain the more accurate solution scheme. In addition, the programming implementation is quite complex, that is, coding the problem first and decoding the problem after finding the optimal solution. The algorithm description of GA is given in [19] and not detailed here briefly.

2.4.2. CSO. CSO is a novel bionic algorithm that fully inherits the characteristics of swarm intelligence optimization. Individual classification and cooperative optimization are utilized in this algorithm. Thus, the optimal solution maximally can be dug and prematureness can be avoided simultaneously. The CSO mimics the hierarchal order and the individual foraging behavior of the chicken swarm. According to the fitness value of the chicken swarm, it can be divided into several groups, each of which consists of one rooster and some hens and chicks. Each individual corrects its position to get a better feeding position according to its own laws of motion, so that its fitness value is as small as possible. Mimicking this chicken wisdom can be a good solution to practical optimization problems. The detailed algorithm description of CSO could be referred to in [20]. 


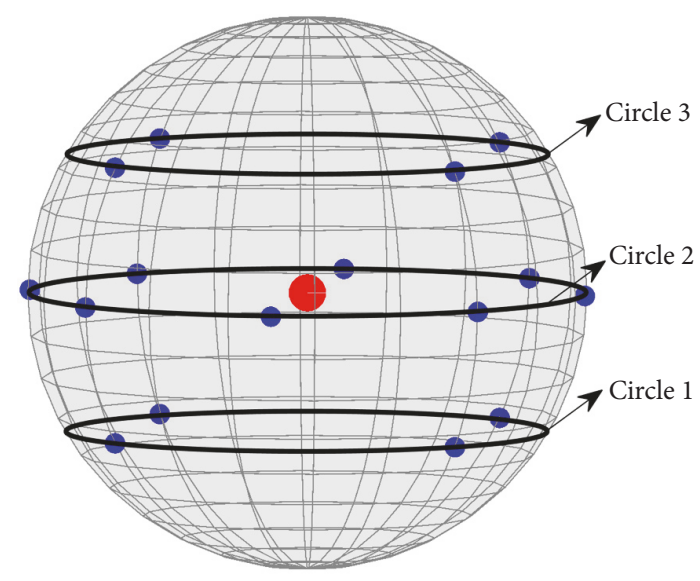

FIGURE 2: An original 3D probe setup with 16 probes. Red mark denotes the center of 3D sphere and blue marks denote the position of probes on the sphere.

2.4.3. ICSO. Considering that the original CSO is easy to fall into local optimum and premature convergence when solving high-dimensional optimization problem, the ICSO algorithm is presented in [21]. The algorithm adds the part of chicks learning from the rooster in chicks' own group to chick's position update equation and introduces the learning factor and inertia weight. Therefore, it is easier to find the global optimal value using ICSO for high-dimensional optimization problem. The algorithm description of ICSO could be found in [21].

\section{Simulation Results}

3.1. Effect of Azimuth Angle of Probe on MTVS. The effect of azimuth angle of probe on MTVS is assessed based on an original 3D probe setup with 16 probes, as shown in Figure 2. The setup has three elevation-separated circles of OTA probes, named circle 1 , circle 2 , and circle 3 , respectively. In the setup, circle 1 , circle 2 , and circle 3 are composed of four, eight, and four probes, with elevation angle of $-30^{\circ}$, $0^{\circ}$, and $+30^{\circ}$, respectively. The probes are placed evenly on three circles where circle 1 and circle 3 are symmetrical with regard to circle 2; that is, there are four probes having the same azimuth angle in three circles, respectively.

In the original setup with 16 probes, the rms error of spatial correlation is presented as a function of test volume size $d$ for two SPS scenarios that can be seen from Figure 3 . The rms monotonically increases within the rms interval of $[0,0.05]$. There is only one value of $d$ when fixing rms $=0.05$, as marked in Figure 3. In addition, the MTVS can be obtained within the given maximum rms error of 0.05 for each SPS scenario, that is, $0.75 \lambda$ and $0.95 \lambda$ for SPS scenarios A and B, respectively. Compared with the MTVS for SPS scenario A, a larger MTVS can be obtained for SPS scenario B.

The effect of azimuth angle of probe on MTVS is assessed by rotating the probe circle horizontally. There are three setup cases of rotating the probe circle considered here. The azimuth angles of probe for each elevation circle for three

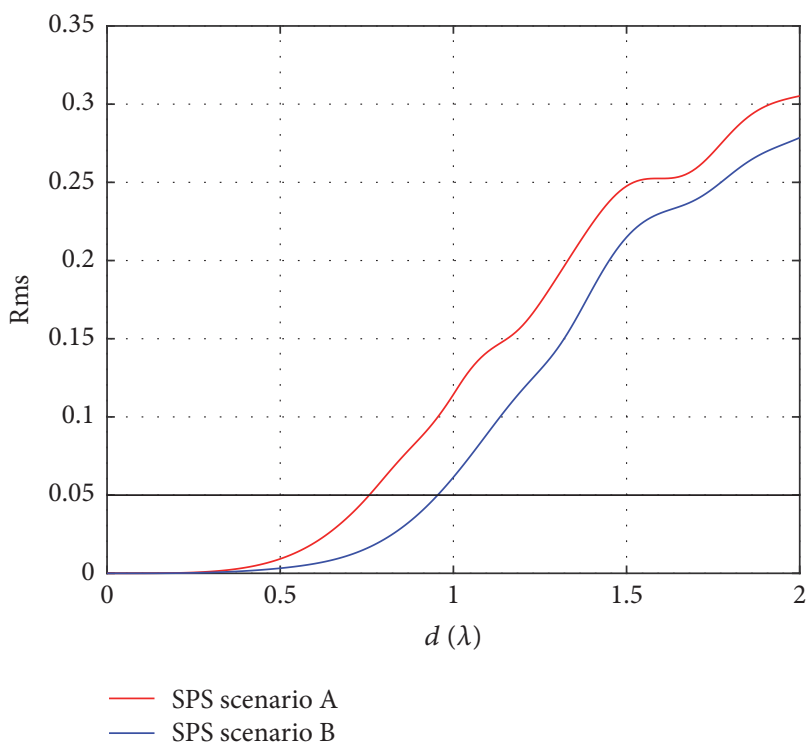

FIGURE 3: Rms error of spatial correlation with test volume size $d$ for two SPS scenarios in the original setup.

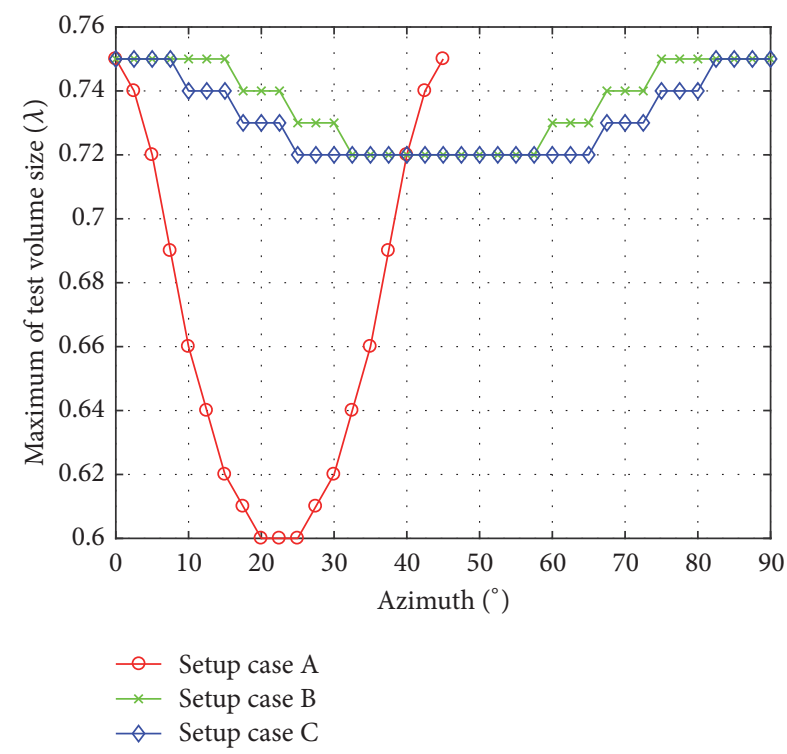

FIGURE 4: MTVS with different rotation angles for three setup cases (cases A, B, and C) employing the SPS scenario A.

setup cases are tabulated in Table 1 . The variables $x, y, z$ represent the rotation angle of probe circle.

Figure 4 displays the MTVS with different rotation angles for three setup cases employing the SPS scenario A. It can be found that the MTVS decreases with probe circle rotating from $0^{\circ}$ to $22.5^{\circ}$, while the MTVS increases as increasing the rotation angle from $22.5^{\circ}$ to $45^{\circ}$ in setup case A. For both setup cases $B$ and $C$, the curves are both symmetrical with regard to rotation angle $45^{\circ}$. Therefore, the largest MTVS of $0.75 \lambda$ is obtained without rotation (i.e., the original setup) for three setup cases employing the SPS scenario A. 
TABLE 1: Azimuth angles of probe for each elevation circle for three setup cases.

\begin{tabular}{|c|c|c|c|}
\hline \multirow{2}{*}{$\begin{array}{l}\text { Setup } \\
\text { case }\end{array}$} & \multicolumn{3}{|c|}{ Probe azimuth angles } \\
\hline & Circle 1 & Circle 2 & Circle 3 \\
\hline $\mathrm{A}$ & $\begin{array}{c}-180^{\circ}+j \cdot 90^{\circ} \\
j \in[1, \ldots, 4]\end{array}$ & $\begin{array}{c}-180^{\circ}+x+j \bullet 45^{\circ}, \\
j \in[1, \ldots, 8] \\
x \in[0, \ldots, 45]\end{array}$ & $\begin{array}{c}-180^{\circ}+j \bullet 90^{\circ} \\
j \in[1, \ldots, 4]\end{array}$ \\
\hline B & $\begin{array}{c}-180^{\circ}+j \cdot 90^{\circ} \\
j \in[1, \ldots, 4]\end{array}$ & $\begin{array}{c}-180^{\circ}+j \bullet 45^{\circ} \\
j \in[1, \ldots, 8]\end{array}$ & $\begin{array}{c}-180^{\circ}+y+j \cdot 90^{\circ}, \\
j \in[1, \ldots, 4] \\
y \in[0, \ldots, 90]\end{array}$ \\
\hline $\mathrm{C}$ & $\begin{array}{c}-180^{\circ}-z+j \cdot 90^{\circ} \\
j \in[1, \ldots, 4] \\
z \in[0, \ldots, 90]\end{array}$ & $\begin{array}{c}-180^{\circ}+j \cdot 45^{\circ} \\
j \in[1, \ldots, 8]\end{array}$ & $\begin{array}{c}-180^{\circ}+z+j \cdot 90^{\circ}, \\
j \in[1, \ldots, 4] \\
z \in[0, \ldots, 90]\end{array}$ \\
\hline
\end{tabular}

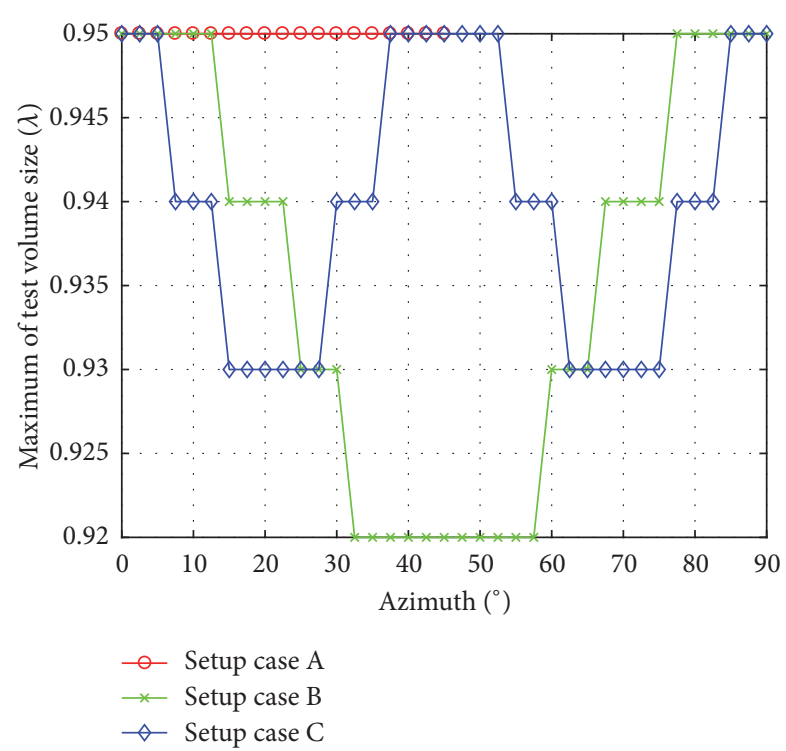

FIGURE 5: MTVS with different rotation angles for three setup cases (cases A, B, and C) employing the SPS scenario B.

The MTVS with variable rotation angle for three setup cases is illustrated in Figure 5 by adopting the SPS scenario B. For setup case A, the MTVS keeps constant with probe circle rotating from $0^{\circ}$ to $45^{\circ}$. The curves are symmetrical with regard to rotation angle $45^{\circ}$ for both setup case B and setup case C. However, the largest MTVS of $0.95 \lambda$ is obtained at rotation angle $45^{\circ}$ besides $0^{\circ}$ for setup case $C$, unlike setup case B.

For setup case C, circle 1 and circle 3 are symmetrical with regard to circle 2 at rotation angle $45^{\circ}$ as well but the azimuth distribution of probes is changed compared with rotation angle $0^{\circ}$. Comparing two SPS scenarios for setup case $\mathrm{C}$, the varied symmetrical setups with different azimuth distributions of probes (i.e., at rotation angle $45^{\circ}$ and $0^{\circ}$ ) have the same MTVS for PAS of uniform distribution, while different for PAS of Laplacian distribution.

3.2. Effect of Elevation Angle of Probe Circle and ES on MTVS. In this part, the effects of elevation angle of probe circle and ES on MTVS are investigated based on the original setup.

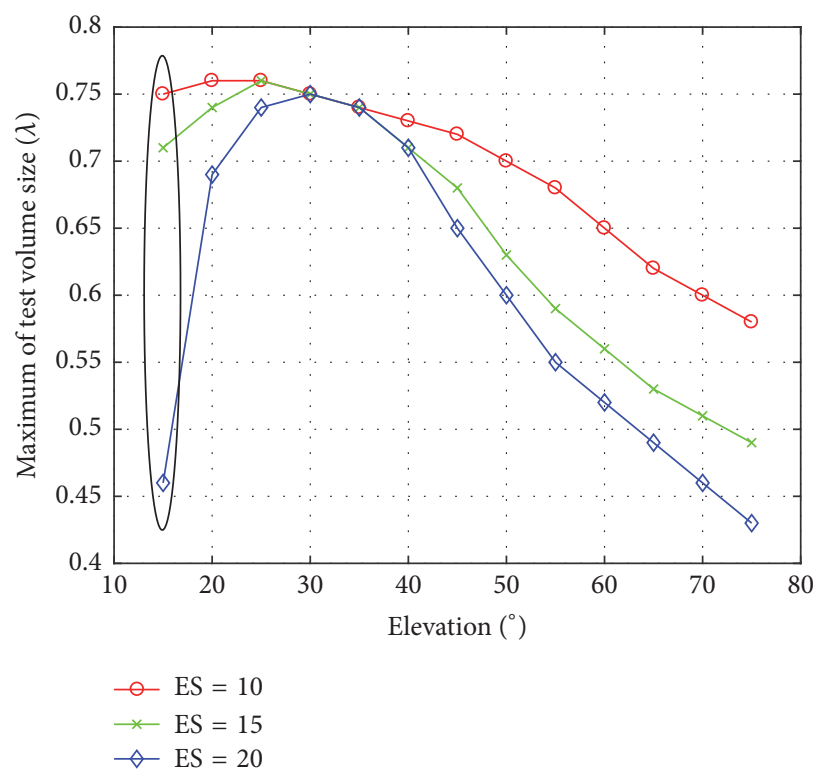

FIgURE 6: MTVS as a function of EPC with different values of ES for SPS scenario A.

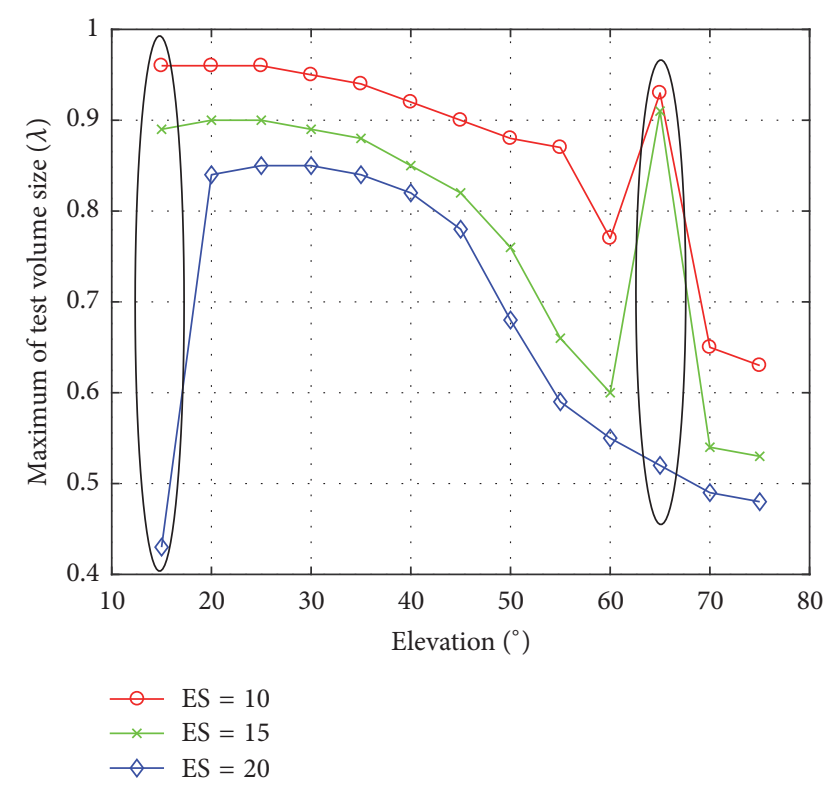

FIGURE 7: MTVS as a function of EPC with different values of ES for SPS scenario B.

The elevation angle of probe circle (EPC) investigated in this paper means the elevation angle of circle 3 mentioned in Section 3.1 where circle 1 and circle 3 are equidistant with regard to circle 2. Considering the practical spherical setup in the anechoic chamber, the EPC is selected from $15^{\circ}$ to $75^{\circ}$ with an interval of $5^{\circ}$ for exploring the effect of EPC. In the practical SPS of 3D channel model, ES is selected from $15^{\circ}$ to $20^{\circ}$ with an interval of $5^{\circ}$ to investigate the effect of ES. Figure 6 plots the MTVS as a function of EPC for SPS scenario A with different values of ES. For SPS scenario B, the MTVS with varying EPC is shown in Figure 7. 
TABLE 2: The parameters of vector representing different test volume sizes for different number of probes.

\begin{tabular}{lccc}
\hline \multirow{2}{*}{ Number of probes } & \multicolumn{3}{c}{ Parameters of vector $(\lambda)$} \\
\hline 8 & $d_{1}$ & $d_{\text {step }}$ & $d_{L}$ \\
12 & 0 & 0.1 & 1 \\
16 & 0 & 0.3 & 1.5 \\
\hline
\end{tabular}

Firstly, the effect of EPC on MTVS is investigated. Fixing $\mathrm{ES}=10^{\circ}$, varied EPCs lead to different MTVSs, as shown in Figures 6 and 7. For SPS scenario A, the function MTVS with $15^{\circ} \leq \mathrm{EPC} \leq 75^{\circ}$ has two same global maxima of $0.76 \lambda$ at EPC $=20^{\circ}$ and $\mathrm{EPC}=25^{\circ}$. For SPS scenario B, the function MTVS with $15^{\circ} \leq \mathrm{EPC} \leq 75^{\circ}$ has three same global maxima of $0.96 \lambda$ at $\mathrm{EPC}=15^{\circ}, \mathrm{EPC}=20^{\circ}$, and $\mathrm{EPC}=25^{\circ}$. Hence, the optimal EPC for the largest MTVS can be found when azimuth angle of probe, the number of probes, and ES are given.

Then the influence of ES on MTVS is studied when fixing $\mathrm{EPC}=30^{\circ}$. The identical MTVS of $0.75 \lambda$ is obtained with three different values of ES for SPS scenario A from Figure 6. As shown in Figure 7, ES of $10^{\circ}$ can achieve the largest MTVS of $0.95 \lambda$ among three different values of ES for SPS scenario $\mathrm{B}$, while ES of $20^{\circ}$ can obtain the smallest one. Hence, the optimal ES for the largest MTVS can be found when azimuth angle of probe, the number of probes, and EPC are given.

Finally, the effects of combining EPC and ES on MTVS are researched. For SPS scenario A, the effect of ES becomes more evident when EPC is not in an interval of $\left[25^{\circ}, 40^{\circ}\right]$. For instance, when setting EPC $=15^{\circ}$, as highlighted in Figure 6 , MTVS is $0.75 \lambda$ with ES $=10^{\circ}, 0.72 \lambda$ with $\mathrm{ES}=15^{\circ}$, and $0.46 \lambda$ with $\mathrm{ES}=20^{\circ}$. However, three different values of ES have the same MTVS at EPC $30^{\circ}$ and $35^{\circ}$. For SPS scenario B, the effect of ES is clear when EPC is $15^{\circ}$ and $65^{\circ}$, as highlighted in Figure 7 . When setting the EPC as $15^{\circ}$, MTVS is $0.96 \lambda$ with $\mathrm{ES}=10^{\circ}, 0.89 \lambda$ with $\mathrm{ES}=15^{\circ}$, and $0.42 \lambda$ with $\mathrm{ES}=20^{\circ}$. When considering EPC of $65^{\circ}$, MTVS is $0.93 \lambda$ with ES $=10^{\circ}, 0.91 \lambda$ with $\mathrm{ES}=15^{\circ}$, and $0.52 \lambda$ with $\mathrm{ES}=20^{\circ}$. The function MTVS with $\mathrm{ES}=10^{\circ}$ and the function MTVS with $\mathrm{ES}=15^{\circ}$ both have a local maximum at $\mathrm{EPC}=65^{\circ}$.

Due to the effects of both EPC and ES on MTVS for two SPS scenarios, it can be found that the EPC for the largest MTVS increases as the value of ES increases. For SPS scenario A, the minimal EPC for the largest MTVS is $10^{\circ}$ larger than ES for each ES. For SPS scenario B, the minimal EPC for the largest MTVS is $5^{\circ}$ larger than ES for each ES. In addition, the MTVS decreases generally along with an augmentation of ES for different EPCs. Therefore, the optimal combination of EPC and ES for the largest MTVS can be acquired for each SPS scenario.

3.3. Effect of Number of Probes on MTVS. The effect of different number of probes in the flexible probe configuration on MTVS is investigated in this part where eight probes, twelve probes, and sixteen probes are considered, respectively. The parameters of vector $\mathbf{d}$ representing variable test volume sizes for different number of probes are summarized in Table 2.
TABLE 3: Test cases considered for algorithm comparison.

\begin{tabular}{lccc}
\hline $\begin{array}{l}\text { Test } \\
\text { case }\end{array}$ & PLOA & $\begin{array}{c}\text { Algorithm parameters } \\
\text { Maximum number of } \\
\text { iterations }\end{array}$ & Population size \\
\hline A & GA & 100 & 50 \\
B & CSO & 100 & 50 \\
C & ICSO & 100 & 50 \\
D & CSO & 200 & 100 \\
\hline
\end{tabular}

TABLE 4: Statistics of the MTVS results for two SPS scenarios and test case A employing different number of probes.

\begin{tabular}{lcc}
\hline $\begin{array}{l}\text { Number of } \\
\text { probes }\end{array}$ & \multicolumn{2}{c}{ MTVS $(\lambda)$} \\
\hline 8 & SPS scenario A & SPS scenario B \\
12 & 0.92 & 0.56 \\
16 & 1.45 & 0.72 \\
\hline
\end{tabular}

TABLE 5: Statistics of the MTVS results for SPS scenario A and the test cases $\mathrm{A}, \mathrm{B}$, and $\mathrm{C}$ employing different number of probes.

\begin{tabular}{lccc}
\hline Number of probes & \multicolumn{3}{c}{ MTVS $(\lambda)$} \\
& Test case A & Test case B & Test case C \\
\hline 8 & 0.92 & 1.02 & 0.89 \\
12 & 1.45 & 1.18 & 1.44 \\
16 & 1.57 & 1.57 & 1.84 \\
\hline
\end{tabular}

Four test cases of PLOA are considered in Table 3, which summarizes two parameters used in the PLOA except the other default parameters.

Considering the test case A firstly, the rms error of spatial correlation is presented as a function of test volume size for two SPS scenarios employing different number of probes that can be found from Figure 8. Fixing rms $=0.05$, statistics of the MTVS results for different number of probes and SPS scenarios are summarized in Table 4 . It can be seen that as the number of probes increases, so does the MTVS. In addition, compared with the MTVS results in the original setup with 16 probes, a larger MTVS can be obtained in the flexible probe configuration for SPS scenario A, while the MTVS is smaller for SPS scenario B. Therefore, the fixed probe configuration is more suitable for channel model with uniform distribution while the flexible probe configuration is more suitable for channel model with nonuniform distribution in terms of obtaining larger MTVSs.

Then considering SPS scenario A, three PLOAs are compared for different number of probes. Figure 9 presents the rms error of spatial correlation as a function of test volume size employing different number of probes for SPS scenario $\mathrm{A}$ and the test cases A, B, and C. The corresponding MTVS results are summarized in Table 5.

CSO can achieve a larger MTVS for eight probes and ICSO can obtain a larger MTVS for sixteen probes 


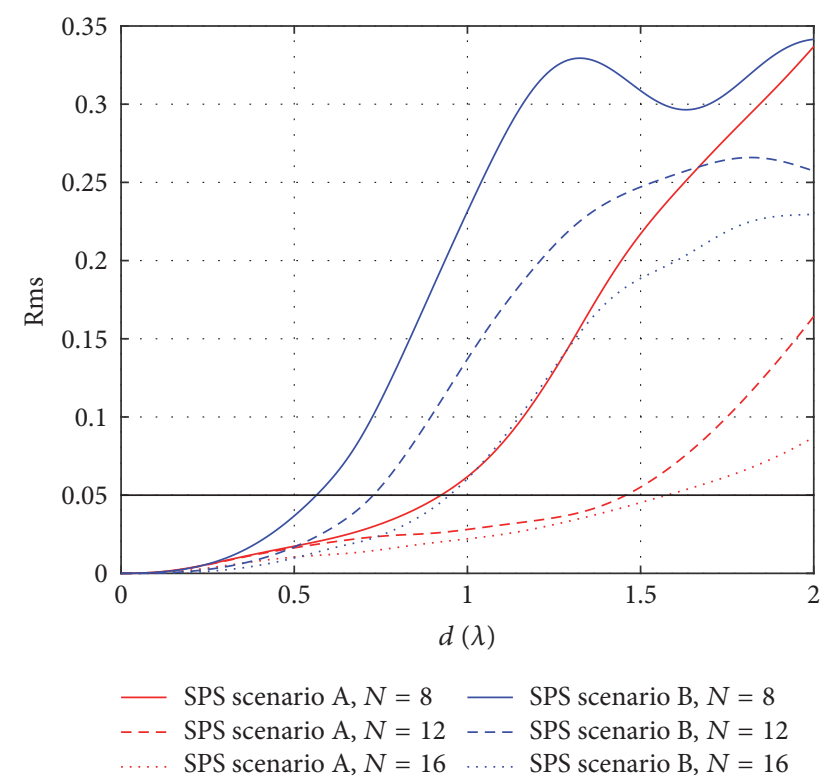

FIGURE 8: Rms error of spatial correlation as a function of test volume size $d$ for two SPS scenarios and test case A employing different number of probes.

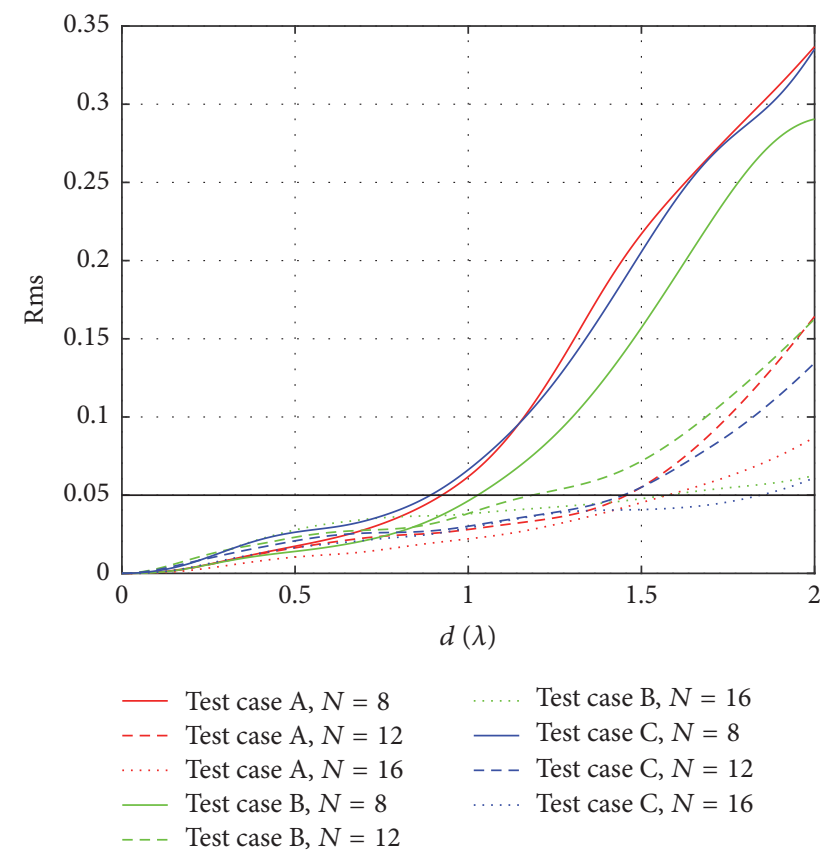

FIGURE 9: Rms error of spatial correlation as a function of test volume size employing different number of probes for SPS scenario $\mathrm{A}$ and the test cases $\mathrm{A}, \mathrm{B}$, and $\mathrm{C}$.

because CSO is suitable for solving low-dimensional optimization problem while ICSO is appropriate for solving highdimensional optimization problem.

Considering CSO finally, different maximum number of iterations and population size are compared for SPS scenario A employing different number of probes. Figure 10 presents the rms error of spatial correlation as a function of test volume
TABLE 6: Statistics of the MTVS results for SPS scenario A and the test cases $\mathrm{B}$ and $\mathrm{D}$ employing different number of probes.

\begin{tabular}{lcc}
\hline $\begin{array}{l}\text { Number of } \\
\text { probes }\end{array}$ & Test case B & MTVS $(\lambda)$ \\
\hline 8 & 1.02 & Test case D \\
12 & 1.18 & 1 \\
16 & 1.57 & 1.55 \\
\hline
\end{tabular}

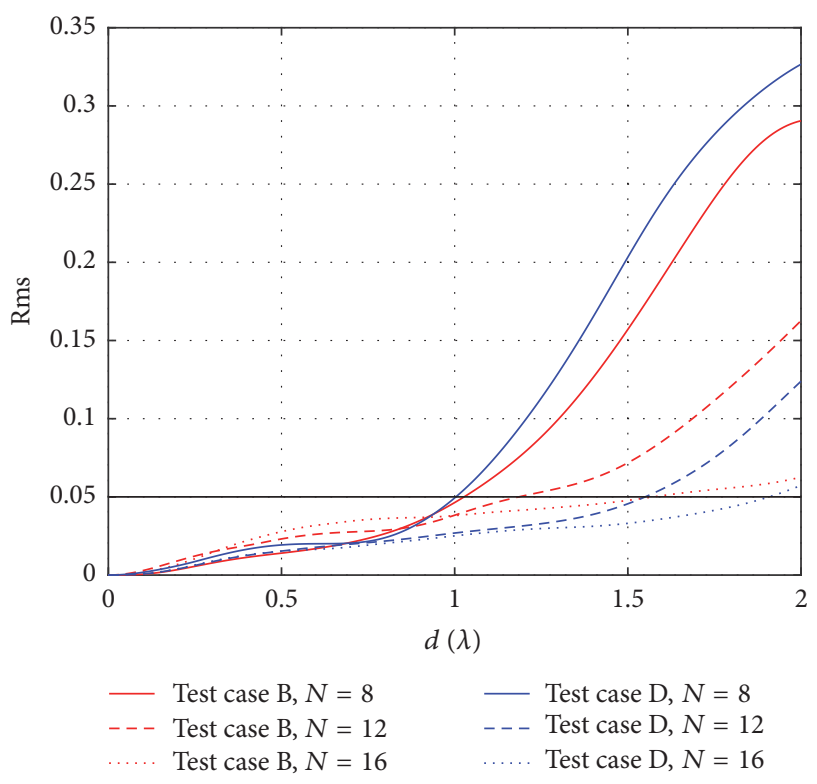

FIGURE 10: Rms error of spatial correlation as a function of test volume size employing different number of probes for SPS scenario $\mathrm{A}$ and the test cases B and D.

size employing different number of probes for SPS scenario A and the test cases $\mathrm{B}$ and D. The corresponding MTVS results are summarized in Table 6 . It can be demonstrated that larger MTVSs can be obtained by increasing the maximum number of iterations and population size.

\section{Conclusion}

This paper presents a method to determine MTVS and investigates the impact of probe configurations on MTVS in order to obtain larger MTVSs. From the simulation results, it can be deduced that symmetrical configuration is optimal for larger MTVSs considering the effect of azimuth angle of probe on MTVS in the fixed probe configuration. Moreover, the optimal combination of EPC and ES for the largest MTVS can be found for each SPS scenario. In the flexible probe configuration, larger MTVSs can be obtained than those in the fixed probe configuration employing the same number of probes for PAS of nonuniform distribution. In addition, larger MTVSs can be achieved when more probes are employed with PLOA and convex optimization. It is obtained that the PLOA solving high-dimensional optimization problem is more suitable for a larger number of probes. The determination and increasing of MTVS are 
meaningful to determine the maximum of DUT size for a given probe configuration and increase the MTVS for MIMO OTA testing.

\section{Conflicts of Interest}

The authors declare that there are no conflicts of interest regarding the publication of this paper.

\section{Acknowledgments}

This work was supported by the National Natural Science Foundations of China (no. 61327806 and no. 61701041) and by the National Key Basic Research Program of China (973 Program) (no. 2014CB339900).

\section{References}

[1] Y. Jing, Z. Wen, H. Kong, S. Duffy, and M. Rumney, "Two-stage over the air (OTA) test method for MIMO device performance evaluation," in Proceedings of the 2011 IEEE International Symposium on Antennas and Propagation and USNC/URSI National Radio Science Meeting, APSURSI 2011, pp. 71-74, Washington, DC, USA, June 2011.

[2] P. K. Agyapong, M. Iwamura, D. Staehle, W. Kiess, and A. Benjebbour, "Design considerations for a $5 \mathrm{G}$ network architecture," IEEE Communications Magazine, vol. 52, no. 11, pp. 65-75, 2014.

[3] "Verification of radiated multi-antenna reception performance of user equipment, 3GGP TR 37.977 V14.1.0," Sep. 2016.

[4] M. Rumney, R. Pirkl, M. H. Landmann, and D. A. SanchezHernandez, "MIMO over-the-air research, development, and testing," International Journal of Antennas and Propagation, vol. 2012, Article ID 467695, 2012.

[5] Y. Okano, K. Kitao, and T. Imai, "Impact of number of probe antennas for MIMO OTA spatial channel emulator , in," in Proceedings of the European Conference on Antennas and Propagation, pp. 1-5, April 2010.

[6] A. Khatun, T. Laitinen, V.-M. Kolmonen, and P. Vainikainen, "Dependence of error level on the number of probes in over-the-air multiprobe test systems," International Journal of Antennas and Propagation, vol. 2012, Article ID 624174, 6 pages, 2012.

[7] L. Foged, A. Scannavini, N. Gross, and J. Estrada, "MIMO OTA testing using a multiprobe system approach," in Proceedings of the European Conference on Antennas and Propagation, pp. 1673-1677, Gothenburg, Sweden, April 2013.

[8] W. Fan, P. Kyosti, S. Fan et al., "3D Channel Model Emulation in a MIMO OTA Setup," in Proceedings of the 2013 IEEE 78th Vehicular Technology Conference (VTC Fall), pp. 1-5, Las Vegas, NV, USA, September 2013.

[9] I. C. Llorente, W. Fan, and G. F. Pedersen, "Mimo ota testing in small multiprobe anechoic chamber setups," IEEE Antennas and Wireless Propagation Letters, vol. 15, pp. 1167-1170, 2016.

[10] P. Kyosti and A. Khatun, "Probe configurations for 3D MIMO Over-the-Air testing," in Proceedings of the 2013 7th European Conference on Antennas and Propagation, EuCAP 2013, pp. 14211425, Gothenburg, Sweden, April 2013.

[11] M. Belhabib, R. D'Errico, and B. Uguen, "Spatial correlation in spherical and cylindrical 3D MIMO Over-The-Air tests setups," in Proceedings of the 10th European Conference on Antennas and Propagation, EuCAP 2016, Davos, Switzerland, April 2016.
[12] P. Kyösti, W. Fan, G. F. Pedersen, and M. Latva-Aho, "On dimensions of OTA setups for massive MIMO base stations radiated testing," IEEE Access, vol. 4, pp. 5971-5981, 2016.

[13] W. Fan, X. Carreño, P. Kyösti, J. Ø. Nielsen, and G. F. Pedersen, "Over-the-air testing of MIMO-capable terminals: evaluation of multiple-antenna systems in realistic multipath propagation environments using an OTA method," IEEE Vehicular Technology Magazine, vol. 10, no. 2, pp. 38-46, 2015.

[14] "Test Plan for 2x2 Downlink MIMO and Transmit Diversity Over-theAir Performance," CTIA Certification, Tech. Rep. Version 1.1, 2016.

[15] M. B. Knudsen and G. F. Pedersen, "Spherical outdoor to indoor power spectrum model at the mobile terminal," IEEE Journal on Selected Areas in Communications, vol. 20, no. 6, pp. 1156-1169, 2002.

[16] R. Vaughan and J. Bach-Anderson, Channels, Propagation and Antennas for Mobile Communications, IET, London, UK, 2003.

[17] S. Boyd and L. Vandenberghe, Convex Optimization, Cambridge University Press, Cambridge, UK, 2004.

[18] H. Gao, W. Wang, Y. Wu, and Y. Liu, “3D Flexible Multiprobe Setups for MIMO OTA Testing," in Proceedings of the 5th International Symposium on EMC, Beijing, China, 2017.

[19] R. L. Haupt and D. H. Werner, Genetic Algorithms in Electromagnetics, Wiley-IEEE Press, Hoboken, NJ, USA, 2007.

[20] X. B. Meng, L. Yu, X. Z. Gao, and H. Z. Zhang, "A new bioinspired algorithm: chicken swarm optimization," in Advances in Swarm Intelligence, vol. 8794 of Lecture Notes in Computer Science, pp. 86-94, Springer International, Brussels, Belgium, 2014.

[21] D. Wu, F. Kong, W. Gao, Y. Shen, and Z. Ji, "Improved chicken swarm optimization," in Proceedings of the 5th Annual IEEE International Conference on Cyber Technology in Automation, Control and Intelligent Systems, IEEE-CYBER 2015, pp. 681-686, China, June 2015. 


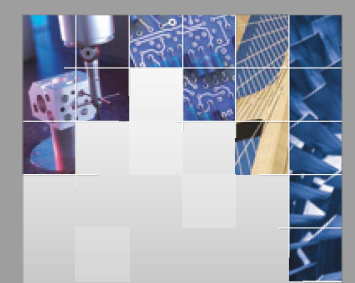

\section{Enfincering}
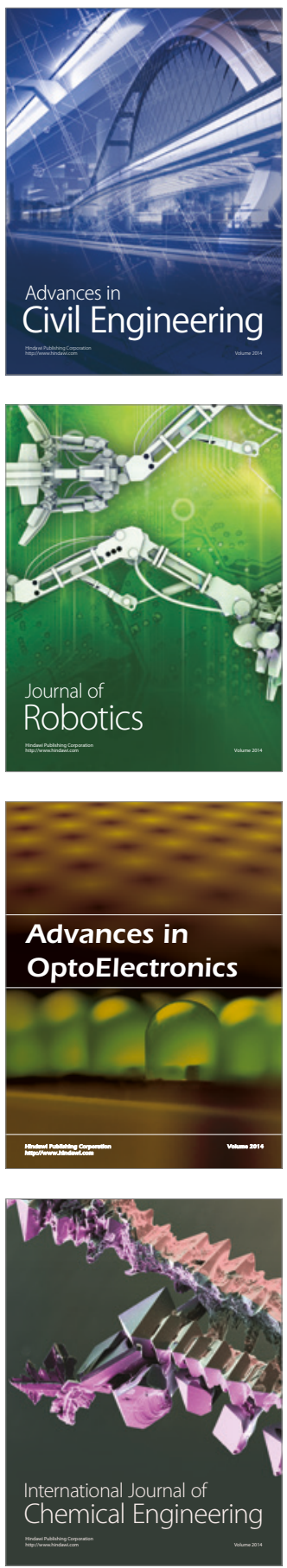

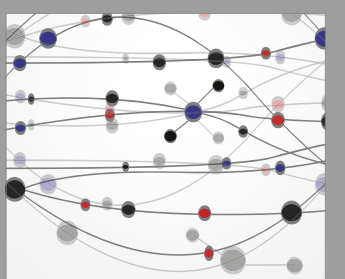

The Scientific World Journal

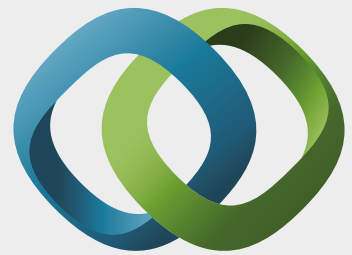

\section{Hindawi}

Submit your manuscripts at

https://www.hindawi.com
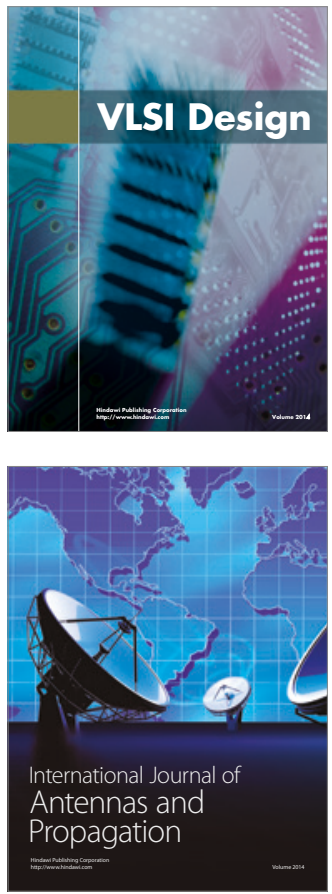

\section{Rotating}

Machinery
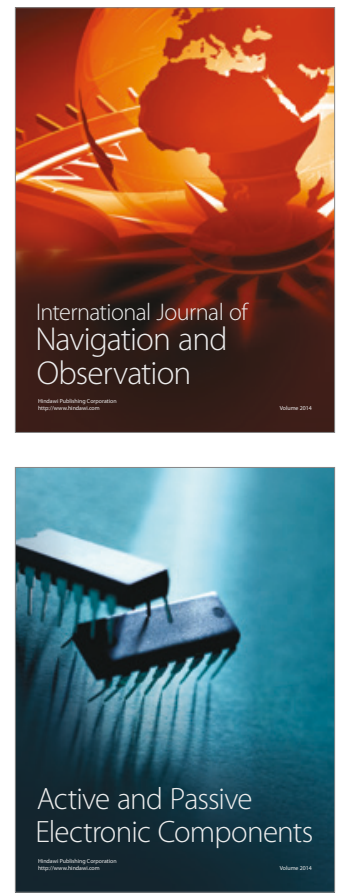
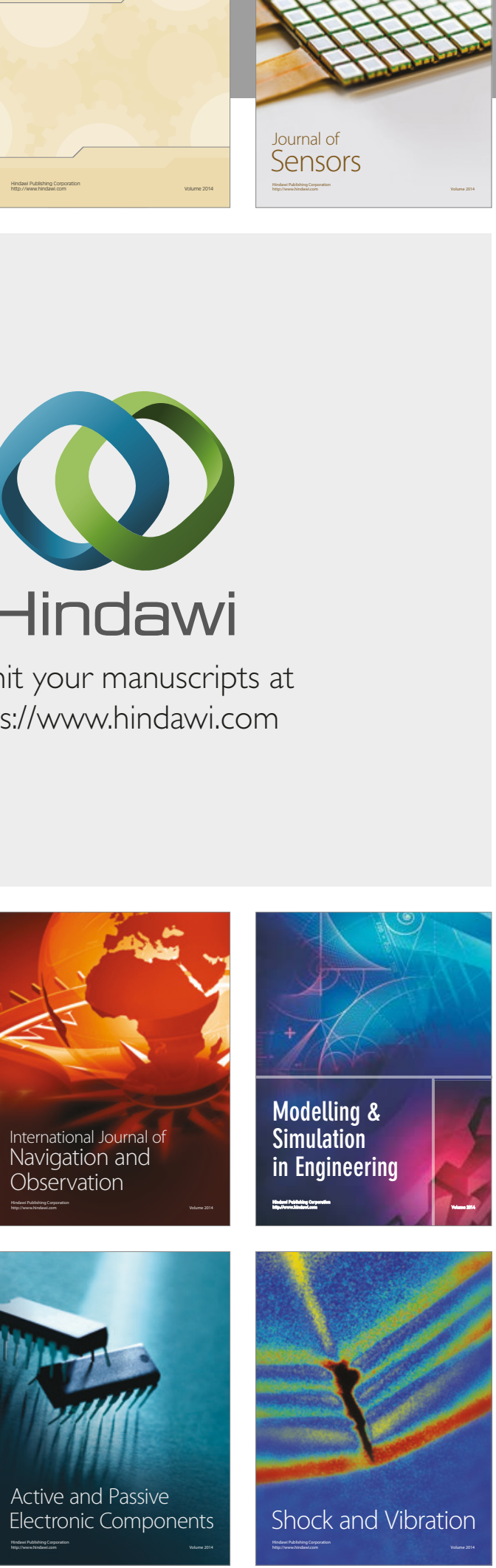
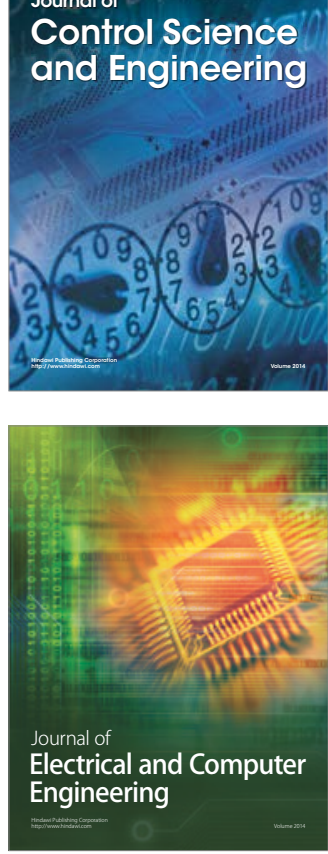

Distributed

Journal of

Control Science

and Engineering
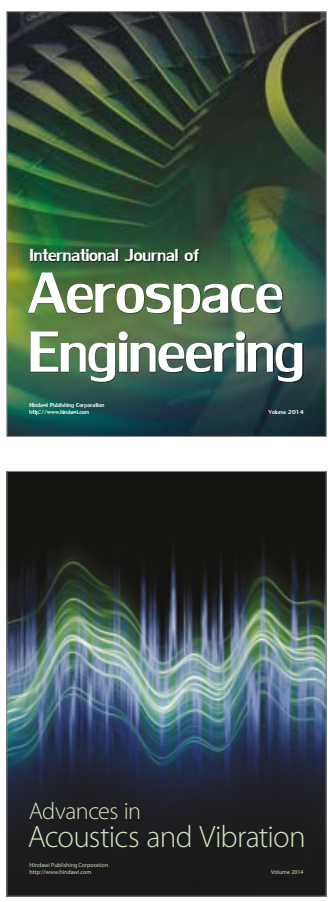

Sensor Networks 